\title{
ON THE STABILITY OF SURFACES OF STRESSED SOLIDS
}

\author{
D. J. SROLOVITZ
}

Department of Materials Science and Engineering, University of Michigan, Ann Arbor, MI 48109, U.S.A

(Received 7 April 1988)

\begin{abstract}
A simple linear stability analysis is presented which demonstrates that the nominally flat surface of an elastically stressed body is unstable with respect to the growth of perturbations with wavelengths greater than a critical wavelength. For a solid, constrained in one dimension and subject to a uniform dilatation, this wavelength scales as $\gamma E / \sigma^{2}$, where $\gamma$ is the surface energy, $E$ is Young's modulus, and $\sigma$ is the nominal stress associated with the constrained dilatation. The maximally unstable mode depends on the manner of matter transport (surface diffusion and evaporation/condensation are considered). The predicted wavelength of the instability is consistent with observations of thin InGaAs films grown on GaAs.
\end{abstract}

Résumé-Nous présentons une analyse simple de stabilité linéaire qui démontre que la surface parfaitement plane d'un corps déformé élastiquement est instable vis à vis de la croissance de perturbations de longueur d'onde supérieure à une valeur critique. Pour un solide, contraint dans une dimension et soumis à une dilatation linéaire, cette longueur d'onde varie comme $\gamma E / \sigma^{2}$, où $\gamma$ est l'énergie superficielle, $E$ est le module d'Young et $\sigma$ est la contrainte nominale associée à la dilatation sous contrainte. Le mode le plus instable dépend de la manière dont s'effectue le transport de matière (diffusion superficielle et évaporation/condensation sont prises en compte). La longueur d'onde prédite pour l'instabilité coïncide avec les observations de films minces de InGaAs déposés sur GaAs.

\begin{abstract}
Zusammenfassung-Es wird eine einfache lineare Stabilitätsanalyse vorgelegt, welche zeight, daß die nominell flache Oberfläche eines elastisch verspannten Festkörpers insofern instabil ist, als Störungen mit Wellenlängen oberhalb einer kritischen wachsen. Bei einem Festkörper, der in einer Dimension eingeschränkt ist und einer gleichmäßigen Dilatation unterliegt, skaliert diese Wellenlänge wie $\gamma E / \sigma^{2}$; hierbei sind $\gamma$ die Oberflächenenergie, $E$ der Elastizitätsmodul und $\sigma$ die mit der eingeschränkten Dilatation zusammenhängende Nominalspannung. Die instabilste Mode hängt von der Art des Materietransportes $\mathrm{ab}$ (betrachtet werden Oberflächendiffusion und Verdampfung/Kondensation). Die vorausgesagten Wellenlängen der Instabilitäten sind mit Beobachtungen an dünnen Filmen auds InGaAs, gezüchtet auf GaAs, verträglich.
\end{abstract}

\section{INTRODUCTION}

It is well known that the development of stresses within solids can lead to morphological changes. For the case of ellipsoidal inclusions subject to transformation strains, Eshelby [1] has shown that the total strain energy of the system varies with the shape of the inclusion. In some cases, needle-like or disklike inclusions are elastically more stable than spherical ones. However, these less symmetrical shapes tend to have a larger surface area than the sphere. In all materials, the surface energy is finite and hence surface tension may oppose the formation of these less symmetrical inclusion shapes. The equilibrium inclusion shape is thus obtained by balancing the clastic energy against the surface energy. Similar considerations are necessary to determine the morphological stability of the surface of a stressed solid.

One case, where both strain energy and surface energy are important, is in thin films. Different modes of the early stages of film growth have been identified (see Ref. [2] for a discussion of these): corresponding to layer (Frank-van der Merwe) growth, island (Volmer-Weber) growth, and layer-then-island (Stranski-Krastanov) growth. Research [2-4] suggests that the various types of growth are dictated by the relative importance of interfacial energy and strain energy contributions. Consider the case of an epitaxially grown film on a substrate with respect to which it is misfitting (i.e., the lattice constant of film $a_{\mathrm{f}}$, and substrate, $a_{\mathrm{s}}$, differ). The strain in the film $\epsilon$ is given by $\left(a_{\mathrm{s}}-a_{\mathrm{f}}\right) / a_{\mathrm{f}}$, where we have assumed that the substrate is much thicker than the film. A small difference in lattice constants can lead to very large stresses since plastic relaxation in very thin films is much morc difficult than in bulk materials. For example, in the absence of plastic relaxation, a $1 \%$ lattice constant mismatch between an InGaAs film and a GaAs substrate can lead to stresses of order $2 \mathrm{GPa}$. When the characteristic dimension of a mate- 


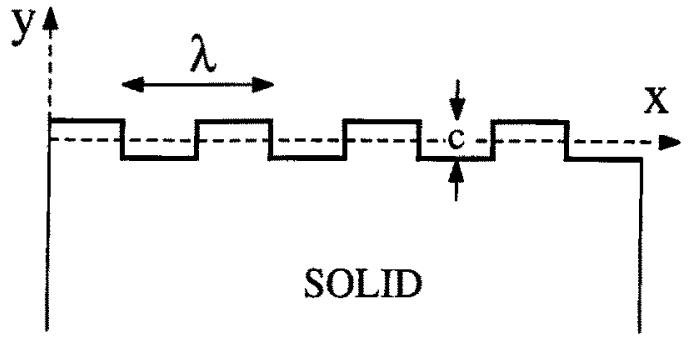

Fig. I. A solid with a square wave surface profile. The surface profile has wavelength $\lambda$ and amplitude $c / 2$.

rial, $r$, is very small, the importance of surface energy, $\gamma$, can be great. In order to make a rough comparison of the magnitudes of the surface and elastic contributions, we compare the elastic stress with the surface tension, $\gamma / r$. For the InGaAs case described above, the elastic stress and the surface tension are roughly equal for $r$ of order $10 \mathrm{~nm}$.

A simple energetic analysis shows the nature of the expected instability. Considering the simple square wave surface morphology of Fig. 1, where the sample is stressed in the $x$-direction. The change in energy in going from the flat surface to that shown in Fig. 1 is roughly

$$
\Delta F=\frac{-\sigma^{2}}{2 E} \frac{c \lambda}{2}+2 \gamma_{c}
$$

where $\sigma$ is the stress in the bulk, $\gamma$ is the surface energy, and $E$ is the modulus and where we have crudely assumed that the stress in the interior of the square protrusions are zero. Equation (1) shows that the formation of this "rough" surface profile lowers the energy of the system provided that the wavelength, $\lambda>8 \gamma E / \sigma^{2}$. Although the above analysis is crude, it demonstrates why the surfaces of stresses bodies may be unstable.

In the present paper, we present a more rigorous kinetic stability argument that demonstrates that flat surfaces bounding elastically stressed solids are unstable with respect to the formation of surface undulations of wavelength greater than a critical wavelength, $\lambda_{c}$. That wavelength, and the maximally unstable wavelength $\left(\lambda_{\mathrm{m}}\right)$, are determined for two types of matter transport: namely, surface diffusion and evaporation-condensation. While neither $\lambda_{\mathrm{c}}$ nor $\lambda_{\mathrm{m}}$ depend on the magnitude of the transport coefficients, the rate at which perturbations of those wavelengths grow does. A comparison between the predicted wavelengths and observations on a thin semiconductor film is made.

\section{ELASTIC ANALYSIS}

In order to investigate the nature of the surface evolution dictated by the competition between surface and elastic energies (see the following section), we first must determine the effect of changes in the surface profile on the elastic field within the solid. We begin by considering the case of a semi-infinite body with an initially flat surface lying along $y=0$ (see Fig. 2). The body is constrained in the $x$-direction such that $\epsilon_{x x}=0$. This initially unstressed solid is then assumed to undergo a hydrostatic transformation strain (i.c. $\epsilon_{x x}=\epsilon_{y y}=\alpha, \epsilon_{x y}=0$ ). Since the surface, normal to the $y$-direction, is traction-free and the system is constrained in the $x$-direction, the following stress state results

$$
\begin{aligned}
& \sigma_{x x}=\sigma=-E \alpha /(1-v) \\
& \sigma_{y y}=\sigma_{x y}=0
\end{aligned}
$$

where $E$ and $v$ are the Young's modulus and the Poisson ratio of the solid, respectively. Note that this stress field is uniform in the solid. Equation (2) will only be satisfied in situations where no plastic relaxation occurs.

If the surface is not flat, as assumed above, the stress field in the solid is non-uniform. Consider the case of a small amplitude sinusoidal surface profile

$$
h(x)=\Delta \sin (k x)
$$

such that $\Delta k \ll 1$. For this surface, the stress field described by equation (2) leads to non-zero surface tractions. In order to obtain the stress field which satisfies the zero traction boundary conditions [for the surface described in equation (3)], we search for a solution in terms of Airy stress functions, $\Phi\left(\nabla^{4} \Phi=0\right)$, of the form

$$
\Phi=\sigma_{y}^{2} / 2+\sum\left(A_{n}+B_{n} y\right) e^{-n k y} \sin (n k x)
$$

where the summation is over all modes $(1 \leqslant n \leqslant \infty)$. However, since we are assuming that $\Delta k \ll 1$, we truncate the series at lowest order

$$
\Phi=\sigma_{y}^{2} / 2+(A+B y) e^{-k y} \sin (k x) .
$$

In terms of equation (5), the stresses are given by

$$
\begin{aligned}
& \sigma_{x x}=\Phi_{y y}=\sigma-k[2 B-(A+B y) k] e^{-k y} \sin (k x) \\
& \sigma_{y y}=\Phi_{x x}=-k^{2}(A+B y) e^{-k v} \sin (k x) \\
& \sigma_{x y}=-\Phi_{x y}=-k[B-(A+B y) k] e^{-k y} \cos (k x) .
\end{aligned}
$$

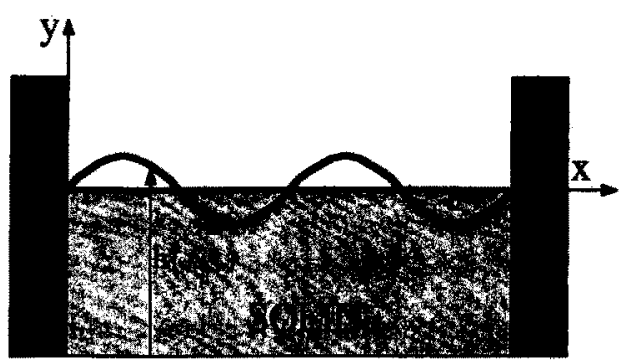

Fig. 2. A solid with a sinusoidal surface profile. The position of the surface $h(x, t)$ is measured with respect to the mean surface height, $y=0$. The solid is elastically constrained in the $\pm x$ direction and is free in the $+y$ direction. 
The stresses contributing to the surface tractions (i.e. those with a normal component) are

$$
\begin{aligned}
& \sigma_{n n}=\sigma_{x x} \sin ^{2} \theta+\sigma_{y y} \cos ^{2} \theta+2 \sigma_{x y} \sin \theta \cos \theta \\
& \sigma_{\tau \tau}=\sigma_{x x} \cos ^{2} \theta+\sigma_{y y} \sin ^{2} \theta+2 \sigma_{x y} \sin \theta \cos \theta \\
& \sigma_{\tau n}=\left(\sigma_{y y}-\sigma_{x x}\right) \sin \theta \cos \theta+\sigma_{x y}\left(\cos ^{2} \theta-\sin ^{2} \theta\right)
\end{aligned}
$$

where $\tan \theta=h_{x}$ and the subscripts $n$ and $\tau$ indicate normal and tangential directions respectively. The requirement that $\sigma_{n n}=\sigma_{\tau n}=0$ along the surface defined by equation ( 3 ) yields

$$
A=0 ; \quad B=E \alpha \Delta /(1-v)=-\sigma \Delta
$$

to lowest order in $\Delta$. The stresses in the solid are thus given by

$$
\begin{aligned}
& \sigma_{x x}=\sigma\left\{1-\Delta k(k y-2) e^{-k y} \sin (k x)\right\} \\
& \sigma_{y y}=\sigma \Delta k^{2} y e^{-k y} \sin (k x) \\
& \sigma_{x y}=\sigma \Delta k^{2}(1-k y) e^{-k y} \cos (k x) .
\end{aligned}
$$

Equation (9) shows that the perturbation of the uniform stress field due to the sinusoidal surface profile decays exponentially into the solid with a decay length proportional to the wavelength of the surface profile.

\section{SURFACE KINETICS}

The evolution of the surface profile is dictated by the chemical potential along the surface. The chemical potential along an interface, $\mu$, is typically written as [5]:

$$
\mu(x)=\mu_{0}+\gamma \Omega \kappa(x)-\sigma_{n n}(x) \Omega
$$

where $\mu_{0}$ is the chemical potential of the equilibrium flat interface bounding an unstressed solid, $\gamma$ is the interfacial tension, $\Omega$ is an atomic volume, and $\kappa$ $\left[=-h_{x x}\left(1+h_{x}^{2}\right)^{-3 / 2}\right]$ is the curvature of the interface. The second term on the right hand side of equation (10) is the surface energy contribution to the chemical potential. The third term accounts for the influence of a stress, normal to the interface, on the emission or absorption of an atom at that interface. This term is, however, not present in the context considered here, wherc the interface is a frec surface and $\sigma_{n n}=0$ is an elastic boundary condition.

If the solid is uniformly stressed, $\mu_{0}$ must be modified to incorporate the strain energy of the solid. This clearly must be the case since the chemical potential is defined as the derivative of the free energy with respect to the number of atoms and the change in energy associated with the addition of an atom to a stressed environment depends on the magnitude and nature of the stress. In order to incorporate this effect directly, the chemical potential described in equation (10) is modified as follows

$$
\begin{aligned}
\mu(x)= & \mu_{0}+\gamma \Omega \kappa(x)+(1 / 2) S_{i j k l} \sigma_{i j}(x) \sigma_{k l}(x) \Omega \\
= & \mu^{*}+\gamma \Omega \kappa(x) \\
& +\left\{\left[\sigma_{\tau \tau}(x)\right]^{2}-\sigma^{2}\right\} \Omega /(2 \mathrm{M})
\end{aligned}
$$

where $\mu^{*}$ is the chemical potential of the flat surface bounding the solid with mean stress $\sigma, S_{i j k l}$ is the compliance matrix, $M$ is the elastic modulus appropriate to the local surface orientation, and repeated indices are summed over. The third term on the right hand side of equation (11) represents the elastic energy of the solid. Since $\sigma_{n n}$ and $\sigma_{\tau n}$ are zero along the surface, the only non-zero surface stress contribution to the implicit summation in equation (11a) is $\sigma_{\tau}$. Since the stresses are generally much less than the modulus, the third term in equation (11) is usually neglected with respect to the $\sigma_{n n}$ term in equation (10) when it is present. For the sinusoidal perturbations analyzed in the previous section [equation (9)], the strain energy term in equation (11b) is given by

$$
\left\{\left[\sigma_{\tau \tau}(x)\right]^{2}-\sigma^{2}\right\} \Omega /(2 M)=\left(2 \Omega k \sigma^{2} / M\right) \sin (k x)
$$

to lowest order in $\Delta k$.

\section{(A) Surface diffusion}

When the surface evolution is controlled by surface diffusion, we may employ the Nernst-Einstein relation to describe the atomic velocity along the surface, $V$

$$
V=-\frac{D_{\mathrm{s}}}{k T} \frac{\partial \mu}{\partial s}
$$

where $D_{s}$ is the surface diffusivity, $k T$ is the thermal energy, and the derivative with respect to $s$ is taken along the surface. This atomic flux leads to a change in surface profile [6]

$$
\frac{\partial h}{\partial t}=\frac{D_{\mathrm{s}} \Omega \delta}{k T} \frac{\partial^{2} \mu}{\partial s^{2}}
$$

where $\delta$ is the number of atoms per unit area. Inserting equations (11) and (12) into equation (14) and assuming $h_{x} \ll 1$ yields

$$
h_{\mathrm{t}}=-C \gamma h_{x x x x}-(C / 2 M)\left\{\left[\sigma_{\tau \tau}(h)\right]^{2}-\sigma^{2}\right\}_{x x}
$$

where $C=D_{\mathrm{s}} \Omega^{2} \delta /(k T)$.

The linear stability of the flat surface bounding the stressed solid may now be analyzed by considering perturbations to the surface. Substituting an initial trial sinusoidal perturbation, $\sin (k x)$, in equation (15), we find it to grow or decay for short time according to

$$
\exp \left\{\left[\left(2 C \sigma^{2} / M\right) k^{3}-C \gamma k^{4}\right] t\right\} .
$$

Thus a band of unstable modes exists with wavelengths $\lambda(=2 \pi / k)>\lambda_{0}\left(=\pi M \gamma / \sigma^{2}\right)$, and with the most unstable mode at $\lambda=\lambda_{\mathrm{m}}=(4 / 3) \lambda_{0}$ (see Fig. 3). These results imply that perturbations of wavelengths smaller than $\lambda_{0}$ are smoothed by surface diffusion, whereas long wavelength perturbations grow unstably. The maximally unstable mode grows as $\exp \left\{(C / 4)(3 / \gamma)^{3}\left[\sigma^{2} /(2 M)\right]^{4}\right\}$. It is interesting to note that the $\lambda_{\mathrm{m}}$ is within a factor of two of that given by the simplistic energy analysis in equation (1). 


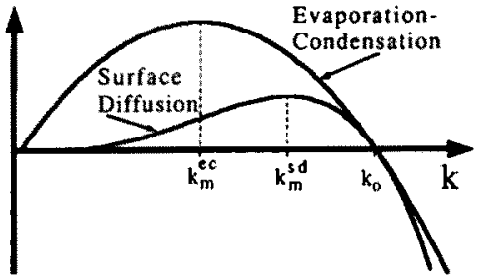

Fig. 3. The exponential growth rate for surface perturbations of wavenumber $k$. The two curves are for surface diffusion controlled growth [equation (16)] and to evaporation condensation controlled growth [equation (18)]. Perturbations with wavenumbers between 0 and $k_{0}$ are unstable. The maximum growth rate (i.e. the maximally unstable mode) occurs for $k=k_{\mathrm{min}}^{\mathrm{w}}$ for surface diffusion controlled growth and for $k=k_{\mathrm{m}}^{\mathrm{ec}}$ when growth is controlled by evaporation and condensation.

\section{(B) Evaporation-condensation}

When the surface evolution is controlled by evaporation from or condensation to a surface (such that there is no net translation of the surface), the differential equation for the evolution of the surface profile has been given by Mullins [6]. Generalizing Mullins' result to include strain energy and only keeping terms to lowest order in $h_{x}^{2}$, we find

$$
h_{1}=C^{\prime} \lambda h_{x x}+C^{\prime} /(2 M)\left\{\left[\sigma_{t r}(h)\right]^{2}-\sigma^{2}\right\}
$$

where $C^{\prime}=P^{*} \Omega^{2}(2 \pi m)^{-1 / 2}(k T)^{-3 / 2}, P^{*}$ is the vapor pressure in equilibrium with the flat surface bounding the stressed body, $m$ is the weight of the molecular species that is evaporating/condensing, and the coefficient of evaporation has been set to unity.

Substituting an initial trial sinusoidal perturbation, $\sin (k x)$, into equation (17), we find it to grow or decay for short time according to

$$
\exp \left\{\left[\left(2 C^{\prime} \sigma^{2} / M\right) k-C^{\prime} \gamma k^{2}\right] t\right\} .
$$

Thus a band of unstable modes exists with wavelengths $\lambda(=2 \pi / k)>\lambda_{0}\left(=\pi M \gamma / \sigma^{2}\right)$, and with the most unstable mode at $\lambda=\lambda_{\mathrm{m}}=2 \lambda_{0}$ (see Fig. 3). These results imply that perturbations of wavelengths smaller that $\lambda_{0}$ are smoothed by the evaporation/condensation process, whereas long wavelength perturbations grow unstably. Note, that while the smallest unstable wavelength is the same as for the surface diffusion case, the wavelength corresponding to the maximally unstable mode is larger by $50 \%$. The maximally unstable mode grows as $\exp \left[\left(C^{\prime} / \gamma\right)\left(\sigma^{2} / M\right)^{2}\right]$.

\section{DISCUSSION}

The results presented in the previous section demonstrate that surfaces of stressed solids are unstable with respect to perturbations of wavelength greater than $\lambda_{0}=\pi M \gamma / \sigma^{2}$. Unfortunately, we have not yet been able to solve the full non-linear surface evolution problem to determine the equilibrium or steady states. Therefore, it is not possible to predict the exact nature of the surface morphology resulting from this instability. However, these results can be applied directly to the problem of a thin film which is misfitting with respect to its substrate. Provided that the film and substrate are not too different elastically (i.e. their moduli are nearly the same) and the interface is coherent (e.g. in epitaxially grown films), then the above results may be directly applied since the maximum amplitude of the surface profile is limited to a few times the film thickness and $k \Delta \ll 1$ is a good approximation.

One such experimental system which has received considerable attention of late is the growth of an InGaAs film on GaAs by means of molecular beam epitaxy. Recent experiments [7] have shown that while a GaAs film can be grown on a GaAs substrate with an atomically flat surface, when some of the Ga is replaced by In the surface becomes rough and an island-like growth process is observed. In changing from pure $\mathrm{GaAs}$ to $\mathrm{In}_{43} \mathrm{Ga}_{57}$ As the lattice parameter increases by $3 \%$ resulting in stresses of order $\sigma=5.8 \mathrm{GPa}$ in the InGaAs film. Recent experimental observations using RHEED and an equilibrium atomistic theory by Berger et al. [7] suggest that the spacing between islands is of order $6 \mathrm{~nm}$ for this case. The atomistic theory is based on a minimization of the total energy of the system with respect to island size and shape by accounting for broken surface bonds and assuming that the first atomic layer of the islands is strained and subsequent layers are at the ideal (unstrained) lattice constant. Since surface diffusion is the likely controlling transport mode in molecular beam epitaxy conditions, we assume $\lambda_{\mathrm{m}}=(4 / 3) \lambda_{0}$. Inserting $\gamma=0.5 \mathrm{~J} / \mathrm{m}^{2}, M=147 \mathrm{GPa}$, and $\sigma=5.8 \mathrm{GPa}$ (which is not readily relaxed due to the extremely small film thickness) into the expression for $\lambda_{\mathrm{m}}$ yields $9 \mathrm{~nm}$. This determination is within approximately $50 \%$ of the reported value. Note, however, that since the theory presented is a continuum theory and the island size is so small, better agreement than this would only be fortuitous.

Can these type of instabilities be observed in macroscopic solids? Inserting the moduli and surface energy associated with pure $\mathrm{Ni}$, and assuming that the stress in the sample is limited to the yield stress, the theory predicts a maximally unstable wavelength of order $5 \mathrm{~mm}$. This length scale is sufficiently large that one would expect observations of such instabilities to be routine. To our knowledge, such observations have not been made. This may be attributable to a number of factors, foremost of which we believe is the small amplitude of the steady state roughness. Unfortunately, since the above theory is simply a stability analysis, it provides essentially no information on the nature of the steady state film profile.

Acknowledgements - The author gratefully acknowledges J. R. Barber's contribution in the elastic analysis presented 
in Section II, K. Chang for discussions which led to this work, and J. Singh, I-W. Chen, and M. Atzmon for useful discussion. This work was supported by a grant from the Exxon Educational Foundation.

\section{REFERENCES}

I. J. D. Eshelby, Proc. R. Soc. A241, 376 (1957); A252, 561 (1959).
2. G. H. Gilmer and M. H. Grabow, J. Metals 39, 19 (1987).

3. R. Bruinsma and A. Zangwill, J. Physique 47, 2055 (1986).

4. J. A. Venables, G. D. T. Spiller and M. Hanbucken, Rep. Prog. Phys. 47, 399 (1984).

5. C. Herring, J. appl. Phys. 21, 437 (1950).

6. W. W. Mullins, J. appl. Phys. 28, 333 (1957).

7. P. R. Berger, K. Chang, P. Bhattacharya, J. Singh and K. K. Bajaj, Appl. Phys. Lett. 53, 684 (1988). 